\title{
Slow cortical rhythms: from single-neuron electrophysiology to whole-brain imaging in vivo
}

\author{
U. OLCESE ${ }^{\prime}$, U. FARAGUNA ${ }^{2,3}$ \\ ' Cognitive and Systems Neuroscience Group, Swammerdam Institute for Life Sciences, University of \\ Amsterdam, Amsterdam, Netherlands; ${ }^{2}$ SonnoLab Pisa, Department of Translational Research and of \\ New Medical and Surgical Technologies, University of Pisa, Pisa, Italy; ${ }^{3}$ Department of Developmental \\ Neuroscience, IRCCS Stella Maris Foundation, Viale del Tirreno 331, 56128 Calambrone, Pisa, Italy
}

\section{A B S T R A C T}

The slow cortical oscillation is the major brain rhythm occurring during sleep, and has been the object of thorough investigation for over thirty years. Despite all these efforts, the function and the neuronal mechanisms behind slow cortical rhythms remain only partially understood. In this review we will provide an overview of the techniques available for the in vivo study of slow cortical oscillations in animal models. Our goal is to provide an up to date resource for the selection of the best experimental strategies to study specific aspects of slow oscillations. We will cover both traditional, population-level electrophysiological approaches (electroencephalography - EEG, local field potentials) as well as more recent techniques, such as two photon calcium imaging and optogenetics. Overall, we believe that new breakthroughs in our understanding of slow cortical rhythms will require the integration of different techniques, to bridge the gap between different spatio-temporal scales and go from a correlative to a causal level of analysis.

Key words

Sleep $\bullet$ Slow oscillation $\bullet$ Slow wave activity $\bullet$ Neurotechnologies $\bullet$ Electrophysiology $\bullet$ Imaging $\bullet$ Optogenetics

\section{Introduction}

Oscillatory rhythms are an emerging property of the central nervous system, and have been surprisingly preserved across brain evolution (Buzsáki et al., 2013). Despite the absence of universal agreement on the breakdown of frequency ranges, several rhythms have been associated with specific brain structures and functions (Steriade, 2006; Buzsáki et al., 2013; Womelsdorf et al., 2014). A notable example is the interplay between theta and gamma rhythms, which mediate memory encoding and recollection in the hippocampus. In this review we will focus on an arbitrary - but well established - slow cortical rhythm, ranging from 0.1 to $4 \mathrm{~Hz}$ (Borbély et al., 1981; Daan et al., 1984; Steriade et al., 2001; Achermann, 2004; Vyazovskiy et al., 2009b; Van Someren et al., 2011). This cellular and network phenomenon is commonly called Slow Cortical Oscillation (SCO), and is the largest electrophysiological entity occurring within the aforementioned frequency window. SCOs represent the most prominent rhythm occurring in the thalamocortical system during non-rapid eye movement (NREM) sleep (Steriade et al., 2001), and have recently also been associated with quiet wakefulness (Sachidhanandam et al., 2013). Moreover, SCOs are homeostatically regulated across the wake-sleep cycle (Borbély et al., 1981; Daan et al., 1984; Achermann, 2004; Leemburg et al., 2010), and their intensity correlates with the overall level of neuronal synchrony (Esser et al., 2007; Vyazovskiy et al., 2008, 2009b). Despite their prominence and highly regulated nature, the function of slow cortical rhythms are still subject of debate. In particular, an ever-increasing amount of evidence links SCOs to sleep-dependent memory consolidation, although the mechanisms remain unclear. 
Specifically, local increase in slow wave activity (SWA, the total EEG power in the SCO frequency range) over motor cortices has been causally implicated in motor learning (Huber et al., 2004, 2006; Marshall et al., 2006; Landsness et al., 2009). This use-dependent increase in SWA, moreover, has been associated with an increase in network synchrony, which in turn can be a function of overall synaptic strength (Marshall et al., 2006; Esser et al., 2007; Huber et al., 2007; Vyazovskiy et al., 2008, 2009b, 2011b; Olcese et al., 2010). Some investigators have hypothesized a role of SCOs in mediating sleepdependent memory consolidation by renormalizing the strength of synaptic connections throughout the cortex (Tononi and Cirelli, 2006, 2014). A competing hypothesis (Mölle and Born, 2011) presents SCOs as grouping events which promote the synchronization of distal brain structure (hippocampus and cortex); the synchronous reactivation of hippocampal and cortical structures during sleep - which has been extensively documented, see e.g. (Euston et al., 2007; Ji and Wilson, 2007) - would then promote a further strengthening of memory traces (Marshall et al., 2006; Rasch et al., 2007).

The physiological relevance of SCOs has made them the object of extensive investigation by many research groups. However, they have remained until now an elusive subject of investigation as their origin and functions still remain not fully understood. The goal of this review is to provide an overview of the most relevant techniques for the investigation of the SCO (see Figure 1A-B). Animal research in particular remains the most powerful approach for the study of the neuronal bases of the brain rhythms; this is especially true for SCOs, as: $i$ ) they involve and are influenced by multiple brain regions (Steriade, 2003), ii) they are modulated by an interplay of intrinsic and extrinsic factors, such as neuromodulators and use of brain structures (Huber et al., 2004; Vyazovskiy et al., 2009b, 2011b), and iii) they play a strong role in memory consolidation and in the regulation of cortical synchrony.

In the next sections we will therefore focus on techniques for in vivo animal investigation, although some methodologies can also be applied to human (e.g. EEG) or to in vitro studies (e.g. patch clamp). Our objective is to provide a resource that might help researchers in the selection of the optimal experimental strategy to study specific aspects of SCOs. After introducing the origin and discovery of SCOs, we will separately cover observational (i.e. recording techniques) and perturbational approaches for the study of slow oscillations.

\section{Discovery}

The first experimental evidence of a cellular event characterized by recurrent transitions between relatively depolarized and hyperpolarized neuronal resting membrane potentials dates back to 1993 (Steriade et al., 1993). In cats under urethane or ketamine anesthesia, Steriade and colleagues first described slow depolarizing envelopes - with superimposed action potentials - followed by longer phases of silent hyperpolarization, in a high proportion of cortical neurons. The same intracellular recordings later showed how this intrinsic property is present also a) during NREM sleep (Steriade et al., 2001), b) in other brain structures such as the thalamus (Steriade et al., 1993), the amygdala (Paré and Gaudreau, 1996; Pape and Driesang, 1998), the cerebellum (Ros et al., 2009), the basal ganglia (Hutchison et al., 2004) and c) in virtually all neurons across the entire thalamocortical system, regardless of their inhibitory and excitatory connections (Timofeev and Chauvette, 2011).

\section{Observational approaches}

\section{Intracellular recordings}

Besides being employed to first describe slow rhythms, intracellular recordings have also been crucial to reveal the cellular bases of SCOs. Intracellular recordings - usually obtained via whole-cell patch clamp - indeed allow to discriminate between the UP and DOWN phases of SCOs (Steriade et al., 2001), corresponding respectively to depolarized and hyperpolarized states. Conversely, extracellular recordings can only reveal whether a neuron is firing action potentials or not - called ON and OFF periods, respectively (Vyazovskiy et al., 2009b). While ON periods necessarily correspond to UP (depolarized) phases, OFF periods can occur even when membrane potentials are relatively depolarized, yet below the threshold for action potential generation. This is a major problem for extracellular studies usually performed within superficial cortical layers, which display very sparse firing activity even when the membrane potential is depolarized (Barth and Poulet, 2012). However, in vivo whole-cell recordings are usually limited to one neuron at a time, and to a duration not longer than 30 minutes. This makes 

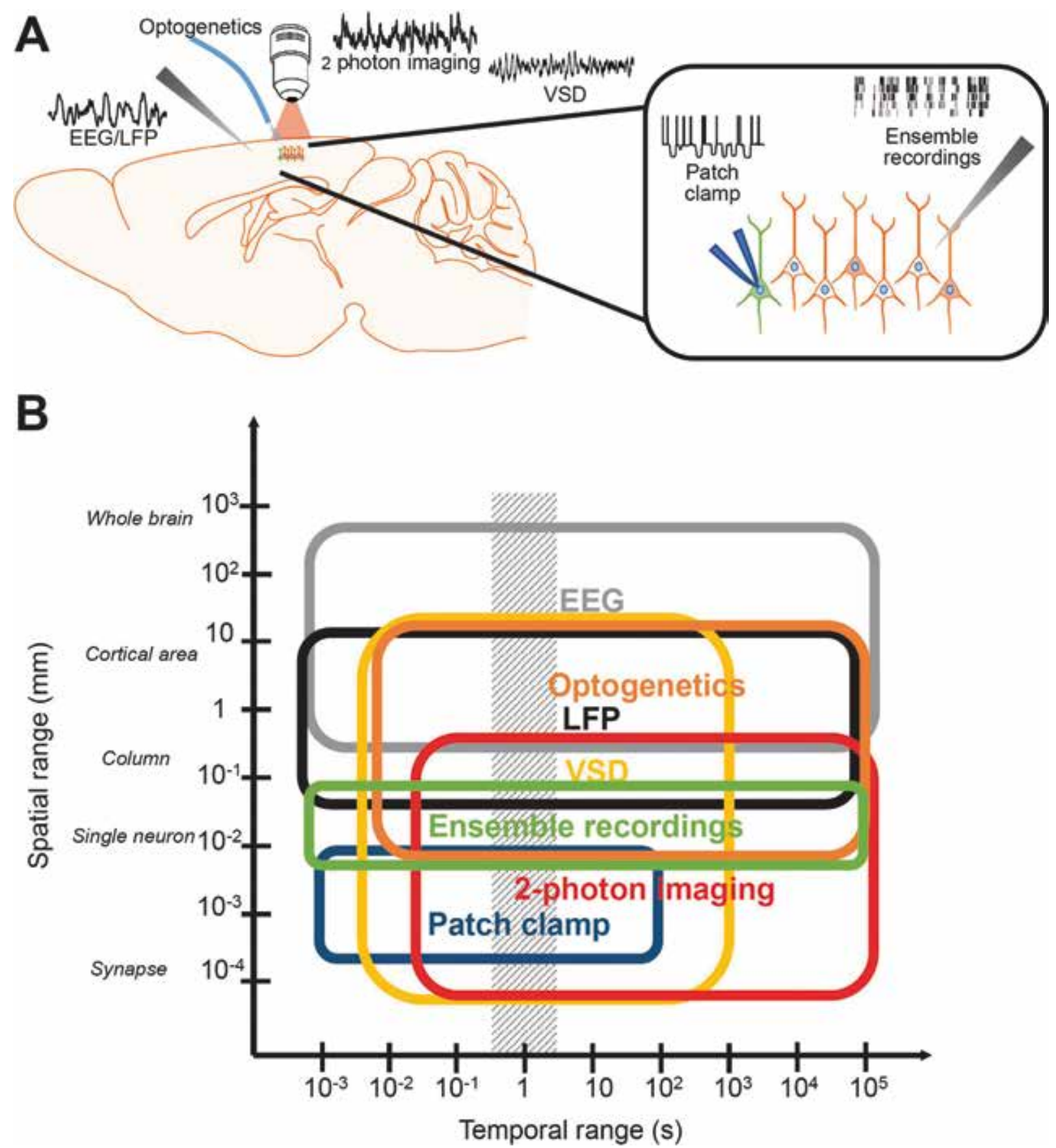

Fig. 1A-B. - Spatio-temporal ranges of techniques for the in vivo investigation of slow cortical rhythms.

Fig. 1A. - Approaches to study SCOs include - from left to right: EEG and LFP recordings (grey triangle), optogenetics (blue optic fiber), two-photon imaging and VSD (objective), patch-clamp recordings (dark blue pipette) and ensemble recordings (grey electrode). While some techniques purely operate at a mesoscale level (EEG, LFP) and others only at a microscopic level (patch-clamp), the integration of different approaches can help researchers span multiple spatio-temporal scales. For example, the use of transgenic animals (neurons of different colors in the right panel) can limit optogenetic interventions to specified neuronal subpopulations, or even to individual neurons, when single-cell electroporation is used (Rancz et al., 2011).

Fig. 1B. - Each of the approaches for studying SCOs enables access to a specific spatio-temporal range. In the graph the bottom-left corner of each rectangle indicates the best spatio-temporal resolution of each technique, while the top-right corner is the largest possible window of observation (e.g. multiple days when employing chronic implants). The shaded area indicates the most important spatio-temporal range for the study of SCOs. Of relevance, recent technological advances have greatly increased the overlap between different techniques. For example, the use of fast scanning methods and genetic calcium indicators makes two-photon imaging suitable to record neuronal activity over days with a resolution of tens of milliseconds. 
them ill-suited for chronic recordings or networklevel analyses.

Until a few years ago, intracellular recordings were mostly performed in anesthetized animals - as even micrometric body movements can lead to the loss of connection between neurons and recording capillaries. This mostly limited in vivo whole-cell patch clamp to the study of anesthesia-related cortical SCOs (e.g. (Haider et al., 2006)). Intriguingly, one outcome of this limitation is that most studies on sensory-evoked intracellular activity in sensory cortices were also performed in anesthetized animals (see for example (Yazaki-Sugiyama et al., 2009; Iurilli et al., 2012; Jacob et al., 2012; Olcese et al., 2013)). Thus, our understanding of how sensory processing occurs during SCOs is deeper than that about sensory-evoked modulation of neural activity during the awake state, despite the larger behavioral relevance of the latter. The introduction of reliable and light-weight head-fixation mechanisms has recently made possible to perform whole-cell recordings in behaving mice and rats, habituated to head-restraint (Sachidhanandam et al., 2013; Guo et al., 2014). This has allowed investigating in more detail the neuronal bases of slow cortical oscillatory activity occurring during natural NREM sleep and quiet wakefulness (Constantinople and Bruno, 2011; Sachidhanandam et al., 2013; Tan et al., 2014).

Another unique opportunity offered by whole-cell recordings is the unambiguous identification of recorded neurons. This can be performed by filling recorded neurons with a dye (e.g. biocytin (Wilson and Sachdev, 2004)). By combining electrophysiological patterns of activity, cell location and the reconstruction of the axonal-dendritic arborization, cell types can be identified and classified (Petilla Interneuron Nomenclature Group et al., 2008). This has been exploited to investigate the role of specific neuronal subpopulations in the generation of SCOs (Steriade et al., 1993, 2001; Constantinople and Bruno, 2011). Integration with imaging techniques, such as two-photon imaging, has allowed targeting recordings to identified neuronal subpopulations, which can for example express fluorescent markers in transgenic animals. Specific interneurons in cortical layer 2-3 (e.g. parvalbumin- and somatostatin-positive), which represent about $20 \%$ of cortical neurons, can now be specifically recorded with high efficiency when neurons are visualized under a two-photon microscope (Komai et al., 2006), further allowing the investigation of different neuronal types across behavioral states (Mateo et al., 2011; Gentet et al., 2012). Although very informative, this approach has a few limitations:

- only a limited number of neurons can be intracellularly monitored simultaneously, significantly limiting any network-scale observation;

- targeted intracellular recordings have been so far limited to superficial cortical layers, although several attempts have been made to extend twophoton imaging to deeper locations (Helmchen and Denk, 2005; Mittmann et al., 2011).

Intracellular recordings have so far provided a unique window on the cellular basis of SCOs. The possibility to integrate intracellular recordings with other novel techniques (imaging and optogenetics) in non-anesthetized rodents is one of the most promising avenues for neuroscience. Its application to the study of SCOs will allow researchers to bridge the gap between cellular and population scale, by providing the means to causally relate the activity of distinct neuronal subpopulations to each phase of the oscillation process, and the emerging behavior.

\section{Extracellular multi-unit recordings}

Extracellular multi-unit (ensemble) recordings are a widely used strategy to investigate SCOs and their neuronal correlates (Vyazovskiy et al., 2012). Several factors have made this technique highly suitable for this research objective: $i$ ) the possibility to perform simultaneous multi-unit and LFP recordings, and therefore directly link population and single-unit features (see e.g. (Vyazovskiy et al., 2009b)); ii) the possibility to record activity from basically any brain structure, and perform simultaneous recordings in multiple brain areas (Ji and Wilson, 2007; Vyazovskiy et al., 2011b); iii) the relative lack of complex instrumentation and experimental skills that are needed, in particular when compared with twophoton calcium imaging and intracellular recordings. The main advantage of extracellular multi-unit recordings is that they allow sampling activity chronically with a high temporal resolution (over $30 \mathrm{kHz}$ ) from a high number of recording sites (commercial devices are available for 256 or more recording channels). Conversely, cell-type identification is not based on anatomical or histological features, but on activityrelated properties, such as action potential waveforms and firing patterns (Barthó et al., 2004; Vyazovskiy et 
al., 2009b; Iurilli et al., 2013). This limits the characterization of neuronal cell types to putative excitatory and inhibitory neurons, the latter mostly represented by fast-spiking interneurons (Barthó et al., 2004).

Extracellular multi-unit recordings - usually performed in conjunction with LFP recordings - have provided many insights into the nature of SCOs. First, while the homeostatic nature of sleep regulation was previously only known at the EEG/LFP level (see e.g. (Achermann, 2004; Leemburg et al., 2010)), ensemble recordings have allowed to discover the neuronal correlates of sleep homeostasis (Vyazovskiy et al., 2009b, 2011b, 2013). Moreover, multi-unit recordings have enabled investigators to discover that the structure of spiking activity during cortical UP states is not only highly organized (Luczak et al., 2007), but strongly dependent on the interplay between hippocampus and cortex during the reactivation of memory traces (Siapas and Wilson, 1998; Euston et al., 2007; Ji and Wilson, 2007). Recently, a significant effort has been placed in understanding the relationship between cortical state and sensory-motor processing (Sakata and Harris, 2012; Luczak et al., 2013; Hall et al., 2014; Pachitariu et al., 2015): these studies have begun to shed light on how the overall oscillatory state affects neural processing.

Extracellular multi-unit recordings have already provided great advances towards understanding cortical SCOs. However, they still have a great potential. Integration with novel transgenic approaches will enable them to gain better specificity concerning specific neuronal sub-populations (Kuki et al., 2015), with unparalleled time-resolution. Moreover, the availability of probes with an ever-increasing number of recording channels will open opportunities for the use of analytical tools from other disciplines (e.g. machine learning, system theory (Safonov et al., 2010)) that will allow to better understand the feature of neuronal activity during sleep.

\section{Local field potential and EEG}

Although their mechanistic descriptions are not yet fully understood, and some degree of controversy is still hovering, the signal recorded by Local Field Potential (LFP) and EEG electrodes is generally believed to reflect the synchronous activity of thousands of synapses neighboring the electrode (Buzsáki et al., 2012; Taub et al., 2014).
Within the cerebral cortex LFP recordings are particularly well suited for studying mesoscale dynamics, such as those occurring within and between cortical columns. Since the first description of the SCO, particular attention has being paid to the phase relationship of oscillations at the LFP level and neuronal activity at the single cell level (Steriade et al., 1993). Studies of anesthetized or sleeping animals have shown that neuronal spike activity is coupled to the phase of the slow oscillation: the neuronal ON period (Vyazovskiy et al., 2009c) - when spiking activity reaches its peak - corresponds to the trough of the LFP slow oscillation. The combination of LFP with intracellular multisite recordings has also offered new insights on the temporal organization of transitions between UP and DOWN states. In a series of papers, Volgushev, Timofeev and collaborators have shown how active (UP) and silent states (DOWN) start almost synchronously, and surprisingly in the cat cortex the onset of silent states is synchronized even more precisely than the onset of activity (Volgushev et al., 2006, 2011; Mukovski et al., 2007; Baranauskas et al., 2010; Lemieux et al., 2015).

Slow waves are the main EEG hallmark of NREM (Note for editors: NREM was already defined earlier) sleep and as such they have been long known, well before the discovery of their neuronal counterpart - here described in the previous paragraphs. Due to its noninvasive nature, EEG studies are particularly suited for human neurophysiological studies, making the investigation of the EEG correlates of SCOs in animal models readily translational. Its limited costs, technical feasibility, compatibility with chronic implants, the well-established and standardized analysis make this approach a robust option for studying the real-time large-scale oscillatory activity occurring across the most superficial cortical layers. As both LFP and EEG have historically been the most widely employed tools to investigate SCOs, several excellent reviews have already discussed the relevance of these methods for studying sleep slow waves significance in animals (Campbell and Tobler, 1984; Steriade, 2003, 2006; Davis et al., 2011; Van Someren et al., 2011; Vyazovskiy et al., 2011a; Vyazovskiy and Faraguna, 2015). For this reason, and for the sake of brevity, we will not further discuss these methods here, and we refer readers to the aforementioned reviews. Nevertheless, it is relevant to point out that both techniques still 
represent the "gold standard" for the investigation of SCOs, and - both on their own and integrated with more novel methodologies - still enable crucial insights in understanding slow cortical rhythms.

\section{Voltage sensitive dyes}

Another approach capable of describing oscillation of the resting membrane potential is based on the fluorescence detected from voltage-sensitive dyes (VSD) (Chemla and Chavane, 2010). Despite its high potentialities (high spatio-temporal resolution, possibility to record from large portions of the cortex), this approach has been only mildly applied to the investigation of cortical slow rhythms. In 2008, Takagaki and others studied the propagation of spontaneous sleep-like waves across the cerebral cortex of rats (Takagaki et al., 2008). With a time resolution of 5-6.7 ms, Mohajerani and colleagues applied VSD to the investigation of cortical oscillations in mice either under anesthesia or recovering from it, describing a high synchronization between hemisphere in both vigilance states (Mohajerani et al., 2010). A promising application of VSD requires the combination with other compatible techniques such as optogenetics (Willadt et al., 2014), although particular care is necessary to properly combine imaging and illumination techniques operating at similar wavelengths.

\section{Two-photon calcium imaging}

Two-photon calcium imaging (Stosiek et al., 2003) represents one of the most powerful and fast evolving innovation of the last decade in in vivo animal neurophysiology. It offers a unique window on neural function by allowing to probe relatively large brain areas (on average $300 \mu \mathrm{m}$ for single field of view), with sub-micrometric resolution, down to the level of individual spines (Chen et al., 2011). Although initially limited in the temporal resolution (at most $10 \mathrm{~Hz}$ ) and in the reachable depth (300-400 $\mu \mathrm{m})$, several innovations have made it a suitable method for fast interrogation of almost any brain structure. The most relevant innovations have been the introduction of genetic calcium indicators (Chen et al., 2013a), fast scanning methods (Grewe et al., 2010), and several approaches to reach deep brain tissue (Helmchen and Denk, 2005).

Two-photon calcium imaging has only recently been applied to the study of the SCOs. One of the major advantages with respect to any other technique for probing neuronal activity is the capacity to allow unambiguous identification and recordings of tens of neurons simultaneously. This has enabled researchers to investigate the spatio-temporal dynamics of UP and DOWN states in vivo in cortical layer $2 / 3$ (Kerr et al., 2005; Bushey et al., 2015). Importantly, the unique spatial resolution of this technique has allowed investigators to discover that the phenomenon of sleep-dependent memory reactivation does not stop at individual neurons, but can be observed in single spines (Chen et al., 2013b). Moreover, twophoton structural imaging has also been employed in conjunction with other techniques, such as electrophysiology and optogenetics, to investigate neural correlates of different behavioral states (Gentet et al., 2012; Polack et al., 2013).

Despite its potential, two-photon calcium imaging has so far not been extensively exploited in investigating SCOs. This is due to the low temporal resolution and weak tissue penetration that has until recently been available in commercial microscopes. The widespread adoption of new techniques (fast genetically encoded indicators for chronic experiments, fast scanners, deep-tissue microscopy) will undoubtedly make this technology far more interesting for sleep researchers. The very same technology applied to in vitro studies has shown an incredible potential to dissect the microscopic dynamics of SCOs (e.g. (Cossart et al., 2003)) and we believe the same will occur in the near future for in vivo studies.

\section{Perturbational approaches}

\section{Electrical stimulation}

Electrical stimulation has historically been the most straightforward strategy to modulate neuronal activity, as it permits fast reversible activation of limited brain regions. However, compared to optogenetics (see the next section), it lacks in spatial resolution and does not enable to selectively activate or silence specific neuronal subpopulation. Nevertheless, electrical stimulation has been and remains a useful tool to probe and manipulate SCOs. Electrical stimulation can be delivered either intra-cortically - usually via bipolar electrodes (Vyazovskiy et al., 2009a) - or trans-cranially - via direct transcranial current stimulation (tDCS) (Marshall et al., 2006). While both 
methods can entrain and boost SCOs (Marshall et al., 2006; Vyazovskiy et al., 2009a; Binder et al., 2014), only intra-cortical current injections are used to probe neural excitability, due to their higher intensity compared to tDCS (Vyazovskiy et al., 2008, 2013). Entrainment of SCOs has been employed to demonstrate a causal role in memory consolidation (Marshall et al., 2006; Chauvette et al., 2012; Binder et al., 2014). Conversely, by probing neural excitability via intra-cortical current injections the homeostatic nature of slow oscillatory activity has been further revealed (Vyazovskiy et al., 2008, 2009a, 2013). Finally, intracortical current injections can be exploited to dissect the contribution of individual neuronal pathways and subtypes to the generation of SCOs (Ushimaru and Kawaguchi, 2015).

\section{Optogenetics}

Optogenetics is probably the most revolutionary neuroscientific technique of the last decade (see (Packer et al., 2013) for a recent comprehensive review). Optogenetics exploits genetically-engineered opsins to modulate the activity of specific neuronal subpopulations. In its most common implementation, viral vectors are injected in the brain region of interest to mediate the expression of channelrhodopsin, a cation channel which opens upon illumination of blue light. In its most advanced forms, channelrhodopsin can drive neuronal activity with millisecond resolution (Gunaydin et al., 2010). Inhibitory opsins such as halorhodopsin and archaerhordopsin can instead hyperpolarize neurons upon illumination with yellow light (Mattis et al., 2012). The use of cre-lox recombination can then be successfully employed to limit opsin expression to specified neuronal subtypes (Madisen et al., 2012).

Optogenetics has allowed neuroscientists to go beyond a correlative level of analysis and investigate causal relationships between neural activity and behavior. In particular, optogenetics has all but replaced electrical stimulation of neural circuits in rodents, as the former enables to achieve incredibly higher spatio-temporal resolutions, down to the possibility of modulating activity in one single spine (Packer et al., 2013). The combination of new opsins, novel transgenic tools and advanced optical technologies make optogenetics and incredibly powerful and versatile tool.

Despite being a relatively recent technique, optogenetics has already been extensively applied to in vivo investigation of SCOs. For this reason, we will here focus on some example applications, which represent both the state-of-the-art and the most promising fields of research. Optogenetics has been employed to modulate the properties of SCOs (Kuki et al., 2013; Stroh et al., 2013) and other sleep-related oscillations, such as spindles (Kim et al., 2012; Barthó et al., 2014): by perturbing natural forms of population activity it was possible to learn insights on their nature and functions. Another promising approach involves modulating the activity of individual neuronal subtypes - such as pyramidal neurons across cortical layers and different types of interneurons - to understand how specific subpopulation of neurons influence the initiation and maintenance of SCOs (Mateo et al., 2011; Beltramo et al., 2013; Sachidhanandam et al., 2013). Finally, optogenetics has been employed to understand the contribution of different brain regions - such as the thalamus - in SCOs (David et al., 2013), how longrange connections can allow cortical regions to modulate the oscillatory state of other areas (Zagha et al., 2013), how the functionalities of specific inter-areal connections varies across behavioral states (Zhang et al., 2014).

While this list represents only an example of the potentialities offered by optogenetics for the study of SCOs, it is nevertheless indicative of the most promising approaches for the coming years: manipulation of population activity to uncover the origin of SCOs, role of individual neuronal subtypes, role of specific brain areas.

\section{Summary}

The mechanisms underpinning SCOs are still uncertain. However, a large array of techniques has become available over the years for the in vivo investigation of slow brain rhythms (Figure 1A-B). Importantly, each of the techniques we have presented still has large potentialities to help achieve breakthroughs on the nature of SCOs, even if some have been in use for over a century (e.g. EEG). The most promising approach - in our opinion - relies on the combination of multiple techniques. For example, the integration of LFP and whole-cell recordings enables investigators to explore - at once - the population and the subcellular level. The addition of optogenetic modulation 
of neural activity can strengthen most experimental paradigms by allowing to explore causal, rather than simply correlative, neuronal mechanisms (see e.g. (Beltramo et al., 2013)). Thanks to the everincreasing affordability and ease of use of complex and powerful techniques such as two photon calcium imaging, the coming years offer an unprecedented opportunity to finally understand the nature of SCOs, which are one of the most fundamental yet still poorly understood rhythms of the brain.

\section{Acknowledgments}

This study was supported by the ARPA Foundation (Pisa, Italy) and the Italian Ministry of Health Grant GR-2011-02348998 to U.F.

\section{References}

Achermann P. The two-process model of sleep regulation revisited. Aviat. Space Environ. Med., 75: A37-43, 2004.

Baranauskas G., Mukovskiy A., Wolf F., Volgushev M. The determinants of the onset dynamics of action potentials in a computational model. Neuroscience, 167: 1070-1090, 2010.

Barth A.L., Poulet J.F.A. Experimental evidence for sparse firing in the neocortex. Trends Neurosci., 35: 345-355, 2012.

Barthó P., Hirase H., Monconduit L., Zugaro M., Harris K.D., Buzsáki G. Characterization of neocortical principal cells and interneurons by network interactions and extracellular features. $J$. Neurophysiol., 92: 600-608, 2004.

Barthó P., Slézia A., Mátyás F., Faradzs-Zade L., Ulbert I., Harris K.D., Acsády L. Ongoing network state controls the length of sleep spindles via inhibitory activity. Neuron, 82: 1367-1379, 2014.

Beltramo R., D’Urso G., Dal Maschio M., Farisello P., Bovetti S., Clovis Y., Lassi G., Tucci V., De Pietri Tonelli D., Fellin T. Layer-specific excitatory circuits differentially control recurrent network dynamics in the neocortex. Nat. Neurosci., 16: 227-234, 2013.

Binder S., Berg K., Gasca F., Lafon B., Parra L.C., Born J., Marshall L. Transcranial slow oscillation stimulation during sleep enhances memory consolidation in rats. Brain Stimulat., 7: 508-515, 2014.
Borbély A.A., Baumann F., Brandeis D., Strauch I., Lehmann D. Sleep deprivation: effect on sleep stages and EEG power density in man. Electroencephalogr. Clin. Neurophysiol., 51: 483-495, 1981.

Bushey D., Tononi G., Cirelli C., Sleep- and wakedependent changes in neuronal activity and reactivity demonstrated in fly neurons using in vivo calcium imaging. Proc. Natl. Acad. Sci. U.S.A., 112: 4785-4790, 2015.

Buzsáki G., Anastassiou C.A., Koch C. The origin of extracellular fields and currents - EEG, ECoG, LFP and spikes. Nat. Rev. Neurosci., 13: 407-420, 2012.

Buzsáki G., Logothetis N., Singer W. Scaling brain size, keeping timing: evolutionary preservation of brain rhythms. Neuron, 80: 751-764, 2013.

Campbell S.S., Tobler I. Animal sleep: a review of sleep duration across phylogeny. Neurosci. Biobehav. Rev., 8: 269-300, 1984.

Chauvette S., Seigneur J., Timofeev I. Sleep oscillations in the thalamocortical system induce long-term neuronal plasticity. Neuron, 75: 1105-1113, 2012.

Chemla S., Chavane F., Voltage-sensitive dye imaging: Technique review and models. J. Physiol. Paris, 104: 40-50, 2010.

Chen T.-W., Wardill T.J., Sun Y., Pulver S.R., Renninger S.L., Baohan A., Schreiter E.R., Kerr R.A., Orger M.B., Jayaraman V., Looger L.L., Svoboda K., Kim D.S. Ultrasensitive fluorescent proteins for imaging neuronal activity. Nature, 499: 295-300, 2013.

Chen X., Leischner U., Rochefort N.L., Nelken I., Konnerth A. Functional mapping of single spines in cortical neurons in vivo. Nature, 475: 501-505, 2011.

Chen X., Rochefort N.L., Sakmann B., Konnerth A. Reactivation of the same synapses during spontaneous up states and sensory stimuli. Cell Rep., 4: 31-39, 2013b.

Constantinople C.M., Bruno R.M. Effects and mechanisms of wakefulness on local cortical networks. Neuron, 69: 1061-1068, 2011.

Cossart R., Aronov D., Yuste R. Attractor dynamics of network UP states in the neocortex. Nature, 423: 283-288, 2003.

Daan S., Beersma D.G., Borbély A.A. Timing of human sleep: recovery process gated by a circadian pacemaker. Am. J. Physiol., 246: R161-183, 1984.

David F., Schmiedt J.T., Taylor H.L., Orban G., Di Giovanni G., Uebele V.N., Renger J.J., Lambert R.C., Leresche N., Crunelli V. Essential thalamic contribution to slow waves of natural sleep. $J$. Neurosci., 33: 19599-19610, 2013. 
Davis C.J., Clinton J.M., Jewett K.A., Zielinski M.R., Krueger J.M. Delta wave power: an independent sleep phenotype or epiphenomenon? J. Clin. Sleep Med., 7: S16-18, 2011.

Esser S.K., Hill S.L., Tononi G. Sleep homeostasis and cortical synchronization: I. Modeling the effects of synaptic strength on sleep slow waves. Sleep, 30: 1617-1630, 2007.

Euston D.R., Tatsuno M., McNaughton B.L. Fastforward playback of recent memory sequences in prefrontal cortex during sleep. Science, 318: 1147 $1150,2007$.

Gentet L.J., Kremer Y., Taniguchi H., Huang Z.J., Staiger J.F., Petersen C.C.H. Unique functional properties of somatostatin-expressing GABAergic neurons in mouse barrel cortex. Nat. Neurosci., 15: 607-612, 2012.

Grewe B.F., Langer D., Kasper H., Kampa B.M., Helmchen F. High-speed in vivo calcium imaging reveals neuronal network activity with near-millisecond precision. Nat. Methods, 7: 399-405, 2010.

Gunaydin L.A., Yizhar O., Berndt A., Sohal V.S., Deisseroth K., Hegemann P. Ultrafast optogenetic control. Nat. Neurosci., 13: 387-392, 2010.

Guo Z.V., Hires S.A., Li N., O'Connor D.H., Komiyama T., Ophir E., Huber D., Bonardi C., Morandell K., Gutnisky D., Peron S., Xu N., Cox J., Svoboda K. Procedures for behavioral experiments in head-fixed mice. PloS One, 9: e88678, 2014.

Haider B., Duque A., Hasenstaub A.R., McCormick D.A. Neocortical network activity in vivo is generated through a dynamic balance of excitation and inhibition. J Neurosci., 26: 4535-4545, 2006.

Hall T.M., de Carvalho F., Jackson A. A common structure underlies low-frequency cortical dynamics in movement, sleep, and sedation. Neuron, 83: 1185-1199, 2014.

Helmchen F., Denk W. Deep tissue two-photon microscopy. Nat. Methods, 2: 932-940, 2005.

Huber R., Esser S.K., Ferrarelli F., Massimini M., Peterson M.J., Tononi G. TMS-induced cortical potentiation during wakefulness locally increases slow wave activity during sleep. PloS One, 2: e276, 2007.

Huber R., Ghilardi M.F., Massimini M., Ferrarelli F., Riedner B.A., Peterson M.J., Tononi G. Arm immobilization causes cortical plastic changes and locally decreases sleep slow wave activity. Nat. Neurosci., 9: 1169-1176, 2006.

Huber R., Ghilardi M.F., Massimini M., Tononi G. Local sleep and learning. Nature, 430: 78-81, 2004.
Hutchison W.D., Dostrovsky J.O., Walters J.R., Courtemanche R., Boraud T., Goldberg J., Brown P. Neuronal oscillations in the basal ganglia and movement disorders: evidence from whole animal and human recordings. J. Neurosci., 24: 92409243, 2004.

Iurilli G., Ghezzi D., Olcese U., Lassi G., Nazzaro C., Tonini R., Tucci V., Benfenati F., Medini P. Sound-driven synaptic inhibition in primary visual cortex. Neuron, 73: 814-828, 2012.

Iurilli G., Olcese U., Medini P. Preserved excitatoryinhibitory balance of cortical synaptic inputs following deprived eye stimulation after a saturating period of monocular deprivation in rats. PloS One, 8: e82044, 2013.

Jacob V., Petreanu L., Wright N., Svoboda K., Fox $\mathrm{K}$. Regular spiking and intrinsic bursting pyramidal cells show orthogonal forms of experiencedependent plasticity in layer $\mathrm{V}$ of barrel cortex. Neuron, 73: 391-404, 2012.

Ji D., Wilson M.A. Coordinated memory replay in the visual cortex and hippocampus during sleep. Nat. Neurosci., 10: 100-107, 2007.

Kerr J.N.D., Greenberg D., Helmchen F. Imaging input and output of neocortical networks in vivo. Proc. Natl. Acad. Sci., U.S.A,, 102: 14063-14068, 2005.

Kim A., Latchoumane C., Lee S., Kim G.B., Cheong E., Augustine G.J., Shin H-S. Optogenetically induced sleep spindle rhythms alter sleep architectures in mice. Proc. Natl. Acad. Sci., U.S.A,, 109: 20673-20678, 2012.

Komai S., Denk W., Osten P., Brecht M., Margrie T.W. Two-photon targeted patching (TPTP) in vivo. Nat. Protoc., 1: 647-652, 2006.

Kuki T., Fujihara K., Miwa H., Tamamaki N., Yanagawa Y., Mushiake H. Contribution of parvalbumin and somatostatin-expressing GABAergic neurons to slow oscillations and the balance in beta-gamma oscillations across cortical layers. Front. Neural Circuits, 9: 6, 2015.

Kuki T., Ohshiro T., Ito S., Ji Z.-G., Fukazawa Y., Matsuzaka Y., Yawo H., Mushiake H. Frequencydependent entrainment of neocortical slow oscillation to repeated optogenetic stimulation in the anesthetized rat. Neurosci. Res., 75: 35-45, 2013.

Landsness E.C., Crupi D., Hulse B.K., Peterson M.J., Huber R., Ansari H., Coen M., Cirelli C., Benca R.M., Ghilardi M.F., Tononi G. Sleep-dependent improvement in visuomotor learning: a causal role for slow waves. Sleep, 32: 1273-1284, 2009. 
Leemburg S., Vyazovskiy V.V., Olcese U., Bassetti C.L., Tononi G., Cirelli C. Sleep homeostasis in the rat is preserved during chronic sleep restriction. Proc. Natl. Acad. Sci. U.S.A,, 107: 15939-15944, 2010.

Lemieux M., Chauvette S., Timofeev I. Neocortical inhibitory activities and long-range afferents contribute to the synchronous onset of silent states of the neocortical slow oscillation. J. Neurophysiol., 113: 768-779, 2015.

Luczak A., Bartho P., Harris K.D. Gating of sensory input by spontaneous cortical activity. J. Neurosci., 33: 1684-1695, 2013.

Luczak A., Barthó P., Marguet S.L., Buzsáki G., Harris K.D. Sequential structure of neocortical spontaneous activity in vivo. Proc. Natl. Acad. Sci. U.S.A, 104: 347-352, 2007.

Madisen L., Mao T., Koch H., Zhuo J., Berenyi A., Fujisawa S., Hsu Y.-W.A., Garcia A.J. 3rd, Gu X., Zanella S., Kidney J., Gu H., Mao Y., Hooks B.M., Boyden E.S., Buzsáki G., Ramirez J.M., Jones A.R., Svoboda K., Han X., Turner E.E., Zeng H. A toolbox of Cre-dependent optogenetic transgenic mice for light-induced activation and silencing. Nat. Neurosci., 15: 793-802, 2012.

Marshall L., Helgadóttir H., Mölle M., Born J. Boosting slow oscillations during sleep potentiates memory. Nature, 444: 610-613, 2006.

Mateo C., Avermann M., Gentet L.J., Zhang F., Deisseroth K., Petersen C.C.H. In vivo optogenetic stimulation of neocortical excitatory neurons drives brain-state-dependent inhibition. Curr. Biol., 21: 1593-1602, 2011.

Mattis J., Tye K.M., Ferenczi E.A., Ramakrishnan C., O'Shea D.J., Prakash R., Gunaydin L.A., Hyun M.., Fenno L.E., Gradinaru V., Yizhar O., Deisseroth K. Principles for applying optogenetic tools derived from direct comparative analysis of microbial opsins. Nat. Methods, 9: 159-172, 2012.

Mittmann W., Wallace D.J., Czubayko U., Herb J.T., Schaefer A.T., Looger L.L., Denk W., Kerr J.N.D. Two-photon calcium imaging of evoked activity from L5 somatosensory neurons in vivo. Nat. Neurosci., 14: 1089-1093, 2011.

Mohajerani M.H., McVea D.A., Fingas M., Murphy T.H. Mirrored Bilateral Slow-Wave Cortical Activity within Local Circuits Revealed by Fast Bihemispheric Voltage-Sensitive Dye Imaging in Anesthetized and Awake Mice. J. Neurosci., 30: 3745-3751, 2010.

Mölle M., Born J. Slow oscillations orchestrating fast oscillations and memory consolidation. Prog. Brain Res., 193: 93-110, 2011.
Mukovski M., Chauvette S., Timofeev I., Volgushev M. Detection of active and silent states in neocortical neurons from the field potential signal during slowwave sleep. Cereb. Cortex, 17: 400-414, 2007.

Olcese U., Esser S.K., Tononi G. Sleep and synaptic renormalization: a computational study. $J$. Neurophysiol., 104: 3476-3493, 2010.

Olcese U., Iurilli G., Medini P., Cellular and synaptic architecture of multisensory integration in the mouse neocortex. Neuron, 79: 579-593, 2013.

Pachitariu M., Lyamzin D.R., Sahani M., Lesica N.A. State-dependent population coding in primary auditory cortex. J. Neurosci., 35: 2058-2073, 2015.

Packer A.M., Roska B., Häusser M. Targeting neurons and photons for optogenetics. Nat. Neurosci., 16: 805-815, 2013.

Pape H.C., Driesang RB. Ionic mechanisms of intrinsic oscillations in neurons of the basolateral amygdaloid complex. J. Neurophysiol., 79: 217-226, 1998.

Paré D., Gaudreau H. Projection cells and interneurons of the lateral and basolateral amygdala: distinct firing patterns and differential relation to theta and delta rhythms in conscious cats. $J$. Neurosci., 16: 3334-3350, 1996.

Petilla Interneuron Nomenclature Group, Ascoli G.A., Alonso-Nanclares L., Anderson S.A., Barrionuevo G., Benavides-Piccione R., Burkhalter A., Buzsáki G., Cauli B., Defelipe J., Fairén A., Feldmeyer D., Fishell G., Fregnac Y., Freund T.F., Gardner D., Gardner E.P., Goldberg J.H., Helmstaedter M., Hestrin S., Karube F., Kisvárday Z.F., Lambolez B., Lewis D.A., Marin O., Markram H., Muñoz A., Packer A., Petersen C.C.H., Rockland K.S., Rossier J., Rudy B., Somogyi P., Staiger J.F., Tamas G., Thomson A.M., Toledo-Rodriguez M., Wang Y., West D.C, Yuste R. Petilla terminology: nomenclature of features of GABAergic interneurons of the cerebral cortex. Nat. Rev. Neurosci., 9: 557-568, 2008.

Polack P.-O., Friedman J., Golshani P. Cellular mechanisms of brain state-dependent gain modulation in visual cortex. Nat. Neurosci., 16: 13311339, 2013.

Rancz E.A., Franks K.M., Schwarz M.K., Pichler B., Schaefer A.T., Margrie T.W. Transfection via whole-cell recording in vivo: bridging singlecell physiology, genetics and connectomics. Nat. Neurosci., 14: 527-532, 2011.

Rasch B., Büchel C., Gais S., Born J. Odor cues during slow-wave sleep prompt declarative memory consolidation. Science, 315: 1426-1429, 2007. 
Ros H., Sachdev R.N.S., Yu Y., Sestan N., McCormick D.A. Neocortical networks entrain neuronal circuits in cerebellar cortex. J. Neurosci., 29: 10309-10320, 2009.

Sachidhanandam S., Sreenivasan V., Kyriakatos A., Kremer Y., Petersen C.C.H. Membrane potential correlates of sensory perception in mouse barrel cortex. Nat. Neurosci., 16: 1671-1677, 2013.

Safonov L.A., Isomura Y., Kang S., Struzik Z.R., Fukai T., Câteau H. Near scale-free dynamics in neural population activity of waking/sleeping rats revealed by multiscale analysis. PloS One, 5: e12869, 2010.

Sakata S., Harris K.D. Laminar-dependent effects of cortical state on auditory cortical spontaneous activity. Front. Neural Circuits, 6: 109, 2012.

Siapas A.G., Wilson M.A., Coordinated interactions between hippocampal ripples and cortical spindles during slow-wave sleep. Neuron, 21: 1123-1128, 1998.

Van Someren E.J.W., Van Der Werf Y.D., Roelfsema P.R., Mansvelder H.D., da Silva F.H.L. Slow brain oscillations of sleep, resting state, and vigilance. Prog. Brain Res., 193: 3-15, 2011.

Steriade M. The corticothalamic system in sleep. Front. Biosci., 8: d878-899, 2003.

Steriade M. Grouping of brain rhythms in corticothalamic systems. Neuroscience, 137: 1087-1106, 2006.

Steriade M., Nuñez A., Amzica F., A novel slow (< $1 \mathrm{~Hz}$ ) oscillation of neocortical neurons in vivo: depolarizing and hyperpolarizing components. $J$ Neurosci., 13: 3252-3265, 1993.

Steriade M., Timofeev I., Grenier F. Natural waking and sleep states: a view from inside neocortical neurons. J. Neurophysiol., 85: 1969-1985, 2001.

Stosiek C., Garaschuk O., Holthoff K., Konnerth A. In vivo two-photon calcium imaging of neuronal networks. Proc. Natl. Acad. Sci. U.S.A., 100: 7319-7324, 2003.

Stroh A., Adelsberger H., Groh A., Rühlmann C., Fischer S., Schierloh A., Deisseroth K., Konnerth A. Making waves: initiation and propagation of corticothalamic $\mathrm{Ca} 2+$ waves in vivo. Neuron, 77: 1136-1150, 2013.

Takagaki K., Zhang C., Wu J.-Y., Lippert M.T. Crossmodal propagation of sensory-evoked and spontaneous activity in the rat neocortex. Neurosci. Lett., 431: 191-196, 2008.

Tan A.Y.Y., Chen Y., Scholl B., Seidemann E., Priebe N.J. Sensory stimulation shifts visual cortex from synchronous to asynchronous states. Nature, 509: 226-229, 2014.
Taub A.H., Lampl I. and Okun M. Local Field Potential, Relationship to Membrane Synaptic Potentials. pp. 1-8. In: Jaeger D., and Jung R. (Eds.) Encyclopedia of Computational Neuroscience. Springer, New York, 2015.

Timofeev I., Chauvette S. Thalamocortical oscillations: local control of EEG slow waves. Curr. Top Med. Chem., 11: 2457-2471, 2011.

Tononi G., Cirelli C. Sleep function and synaptic homeostasis. Sleep Med. Rev., 10: 49-62, 2006.

Tononi G., Cirelli C. Sleep and the price of plasticity: from synaptic and cellular homeostasis to memory consolidation and integration. Neuron, 81: 12-34, 2014.

Ushimaru M., Kawaguchi Y. Temporal Structure of Neuronal Activity among Cortical Neuron Subtypes during Slow Oscillations in Anesthetized Rats. J. Neurosci., 35: 11988-12001, 2015.

Volgushev M., Chauvette S., Mukovski M., Timofeev I. Precise long-range synchronization of activity and silence in neocortical neurons during slowwave oscillations [corrected]. J. Neurosci., 26: 5665-5672, 2006.

Volgushev M., Chauvette S., Timofeev I. Long-range correlation of the membrane potential in neocortical neurons during slow oscillation. Prog. Brain Res., 193: 181-199, 2011.

Vyazovskiy V.V., Cirelli C., Pfister-Genskow M., Faraguna U., Tononi G. Molecular and electrophysiological evidence for net synaptic potentiation in wake and depression in sleep. Nat. Neurosci., 11: 200-208, 2008.

Vyazovskiy V.V., Cirelli C., Tononi G. Electrophysiological correlates of sleep homeostasis in freely behaving rats. Prog. Brain Res., 193: 17-38, 2011.

Vyazovskiy V.V., Faraguna U. Sleep and synaptic homeostasis. Curr. Top. Behav. Neurosci., 25: 91-121, 2015.

Vyazovskiy V.V., Faraguna U., Cirelli C., Tononi G. Triggering slow waves during NREM sleep in the rat by intracortical electrical stimulation: effects of sleep/wake history and background activity. $J$. Neurophysiol., 101: 1921-1931, 2009.

Vyazovskiy V.V., Olcese U., Cirelli C., Tononi G. Prolonged wakefulness alters neuronal responsiveness to local electrical stimulation of the neocortex in awake rats. J. Sleep Res., 22: 264-271, 2013.

Vyazovskiy V.V., Olcese U., Hanlon E.C., Nir Y., Cirelli C., Tononi G. Local sleep in awake rats. Nature, 472: 443-447, 2011. 
Vyazovskiy V.V., Olcese U., Lazimy Y.M., Faraguna U., Esser S.K., Williams J.C., Cirelli C., Tononi G. Cortical firing and sleep homeostasis. Neuron, 63: 865-878, 2009.

Vyazovskiy V.V., Olcese U., Tononi G. Investigating Sleep Homeostasis with Extracellular Recording of Multiunit Activity from the Neocortex in Freely Behaving Rats. pp. 237-258. In Fellin T., and Halassa M. (Eds.) Neuronal Network Analysis. Berlin, Springer, 2015.

Willadt S., Canepari M., Yan P., Loew L.M., Vogt K.E. Combined optogenetics and voltage sensitive dye imaging at single cell resolution. Front. Cell Neurosci., 8: 311, 2014.

Wilson C.J., Sachdev R.N.S. Intracellular and juxtacellular staining with biocytin. Curr. Protoc. Neurosci., 1: 1-12, 2004.
Womelsdorf T., Valiante T.A., Sahin N.T., Miller K.J., Tiesinga P. Dynamic circuit motifs underlying rhythmic gain control, gating and integration. Nat. Neurosci., 17: 1031-1039, 2014.

Yazaki-Sugiyama Y., Kang S., Câteau H., Fukai T., Hensch T.K. Bidirectional plasticity in fast-spiking GABA circuits by visual experience. Nature, 462: 218-221, 2009.

Zagha E., Casale A.E., Sachdev R.N.S., McGinley M.J., McCormick D.A., Motor cortex feedback influences sensory processing by modulating network state. Neuron, 79: 567-578, 2013.

Zhang S., Xu M., Kamigaki T., Hoang Do J.P., Chang W.-C., Jenvay S., Miyamichi K., Luo L., Dan Y. Selective attention. Long-range and local circuits for top-down modulation of visual cortex processing. Science, 345: 660-665, 2014. 